\title{
Coordination of High Gamma Activity in Anterior Cingulate and Lateral Prefrontal Cortical Areas during Adaptation
}

\author{
Marie Rothé, ${ }^{1,2}$ René Quilodran, ${ }^{1,2}$ Jérôme Sallet, ${ }^{1,2}$ and Emmanuel Procyk ${ }^{1,2}$ \\ ${ }^{1}$ Inserm U846, Institut Cellule Souche et Cerveau, 69500 Bron, France, and ${ }^{2}$ Université de Lyon, 69003 Lyon, France
}

\begin{abstract}
The anterior cingulate cortex (ACC) and the lateral prefrontal cortex (LPFC) process complementary information for planning and evaluating behavior. This suggests at least that processes in these two areas are coordinated during behavioral adaptation. We analyzed local field potentials recorded in both regions in two monkeys performing a problem-solving task that alternated exploration and repetitive behaviors with the specific prediction that neural activity should reveal interareal coordination mainly during exploration. Both areas showed increased high gamma power after errors in exploration and after rewards in exploitation. We found that high gamma $(60-140 \mathrm{~Hz})$ power increases in ACC were followed by a later increase in LPFC only after negative feedback (errors) or first positive feedback (correct) during the exploration period. The difference in latencies between the two structures disappeared in repetition period. Simultaneous recordings revealed correlations between high gamma power in the two areas around feedback; however, correlations were observed in both exploration and repetition. In contrast, postfeedback beta $(10-20 \mathrm{~Hz})$ power in ACC and LPFC correlated more frequently during repetition. Together, our data suggest that the coordination between ACC and LPFC activity is expressed during adaptive as well as stable behavioral periods but with different modes depending on the behavioral period.
\end{abstract}

\section{Introduction}

Higher cognitive functions rely on the coordination of brain structures and in particular of dissociated frontal regions. Several frontal areas process common or complementary information for planning and adapting behavior. The anterior cingulate cortex (ACC) and the lateral prefrontal cortex (LPFC) represent different reward-related variables and contribute to the control and temporal organization of behavior (Procyk et al., 2000; Averbeck et al., 2002; Matsumoto et al., 2003; Amiez et al., 2006; Averbeck et al., 2006; Procyk and Goldman-Rakic, 2006; Kennerley et al., 2009). Similarity of response suggests common or coherent functioning, and differences in selectivity strength for spatial information, choice, or reward and action values support functional specificities (Seo and Lee, 2008; Wallis and Kennerley, 2010). In addition, ACC and LPFC coexpress different states of activity during

Received Feb. 25, 2011; revised May 20, 2011; accepted June 10, 2011.

Author contributions: E.P. designed research; M.R., R.Q., J.S., and E.P. performed research; M.R. analyzed data; M.R., R.Q., J.S., and E.P. wrote the paper.

This work was supported by the Fyssen Foundation, NRJ Foundation, Agence Nationale de la Recherche (ANR JCJC 06), and Région Rhône-Alpes projet (ible. M.R. and J.S. were funded by Ministère de l'Education et de la Recherche. R.Q. was funded by Facultad de Medicina Universidad de Valparaíso, proyecto MECESUP UVA-106. M.R., R.Q., and J.S. were also funded by Fondation pour la Recherche Médicale. We thank Dr. C.R.E. Wilson for careful reading of the initial manuscript.

The authors declare no competing financial interests.

Correspondence should be addressed to either Marie Rothé or Emmanuel Procyk, Inserm U846, Institut Cellule Souche et Cerveau, 18 avenue du doyen Lépine, 69500 Bron, France, E-mail: marie.rothe@gmail.com or emmanuel.procyk@inserm.fr.

R. Quilodran's present address: Escuela de Medicina, Departamento de Pre-clinicas, Universitad de Valparaiso, Valparaiso, Chile.

J. Sallet's present address: Decision and Action Laboratory, Department of Experimental Psychology, University of Oxford, Oxford, UK.

DOI:10.1523/JNEUROSCI.1016-11.2011

Copyright $\odot 2011$ the authors $\quad 0270-6474 / 11 / 3111110-08 \$ 15.00 / 0$ exploration versus exploitation of rewards (Landmann et al., 2007; Procyk et al., 2008). How these areas collaborate for generating coherent behavior is a central issue.

A variety of mechanisms based on relations between frontal medial cortical evaluative and lateral frontal controlling structures have been proposed to account for rapid behavioral adaptation (Dehaene et al., 1998; Botvinick et al., 2001; Aston-Jones and Cohen, 2005; Koechlin and Hyafil, 2007). For most theoretical models, some product of performance monitoring (conflict detection, error or reward signals) is involved in the regulation of control. For example, evaluative signals from ACC would trigger increased control by LPFC. This relates to the important role of ACC in retroactive switching and in particular in the behavioral reactions to negative feedback (Hikosaka and Isoda, 2010). Note, however, that $\mathrm{AMRI}$ studies investigating whether ACC signals predict changes in LPFC during specific adaptations (e.g., after conflict or error detection) remain inconsistent (Kerns et al., 2004; Fassbender et al., 2009; Hyafil et al., 2009).

Recent research suggests neurobiological mechanisms by which ACC and LPFC could interact (Medalla and Barbas, 2009), but neurophysiological data are scarce. Several neurophysiological markers might be used to study corticocortical interactions. Frontal low rhythm synchronizations have been observed during cognitive tasks (Tsujimoto et al., 2006; Cavanagh et al., 2009). Such oscillations might also be the vehicle to coordinate high gamma oscillations and hence unit firing in distant areas (Sirota et al., 2008). High gamma activity reflects local information processing and conveys task-relevant information (Pesaran et al., 2002; Belitski et al., 2008). High gamma power of ACC local field potentials (LFPs) reflects feedback that is behaviorally relevant (Quilodran et al., 2008). One possibility is that such feedback-related signals are sent to or shared with LPFC to implement adaptation. 
A Lever

Delay

(2s)

ON

FP Off fixation

GO

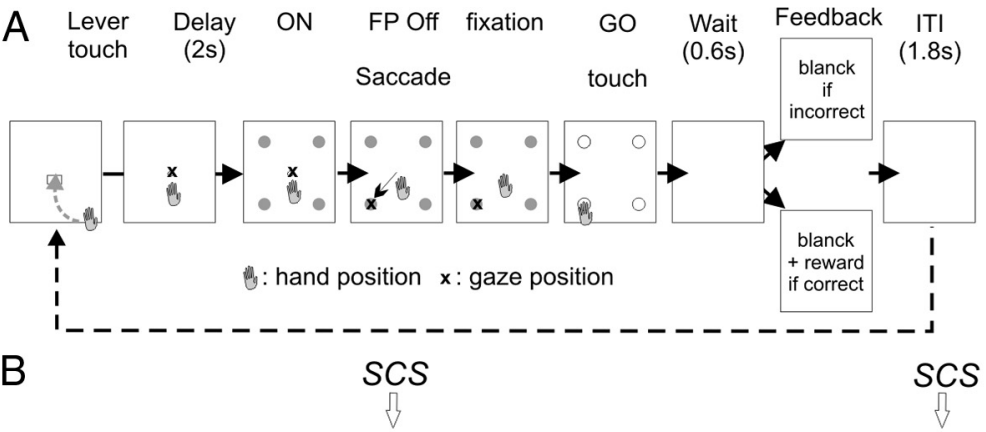

B

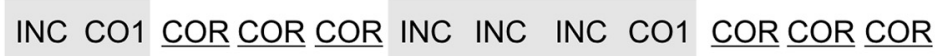
Search Repetition Search Repetition

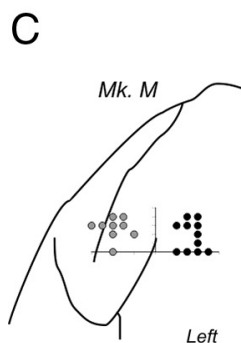
Anterior

Lef

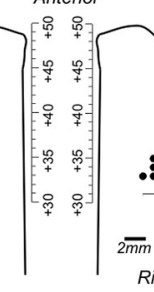

Right

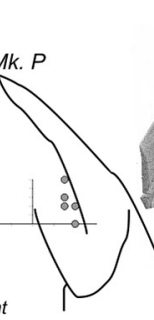

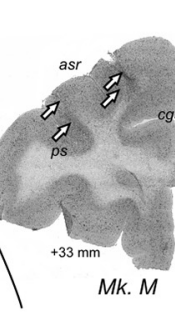

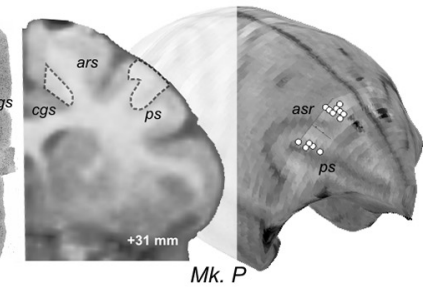

Figure 1. Behavioral task and recording sites. The animal has to search by trial and error for the correct target. $A$, Description of trial events. A trial starts with a touch on the lever and onset of central fixation spot. After a delay period with eye fixation on the central spot, all four targets switch on and the animal makes a saccade toward and touches one of them. All targets switch off, and the feedback is given (no reward: negative; reward: positive). The following trial starts after an intertrial interval (ITI). B, Example of successive problems. In the first problem, the monkey discovers the solution in two trials (search period). After discovery, the animal is allowed to repeat the response (repetition period) (INC, incorrect; $\mathrm{CO1}$, first correct; $\mathrm{COR}$, correct in repetition). A signal to change (SCS) indicates a new problem. $C$, Location of recording sites for the two monkeys $M$ and $P$. The diagrams on the left represent the locations of penetrating sites to target LPFC (gray disks) and ACC (in black disks) in a common stereotaxic space. In the center, a histological section showing electrode tracks (indicated by arrows) targeting LPFC and ACC. On the right, MRI slice and 3D reconstruction for monkey $P$. Reconstruction of penetration sites are shown on the surface of the $3 D$ volume.

Here we compared recordings in ACC and LPFC in monkeys performing a task requiring rapid adaptation and hence a putative coordination between the two areas. By analyzing high gamma activity, we tested three specific predictions: (1) ACC and LPFC should show increased activity after outcomes that induce adjustments in cognitive control (e.g., after negative feedback), (2) ACC feedback-related activity after errors should precede LPFC activity, and (3) power of gamma oscillations simultaneously recorded in both structures is correlated.

\section{Materials and Methods}

Housing, surgical, electrophysiological, and histological procedures were performed in accordance with the European Community Council Directive (1986) (Ministère de l'Agriculture et de la Forêt, Commission nationale de l'expérimentation animale) and Direction Départementale des Services Vétérinaires (Lyon, France). Each animal was seated in a primate chair (Crist Instrument) within arm's reach of a tangent touch screen (Microtouch System; 3M) coupled to a TV monitor. In the front panel of the chair, an opening allowed the monkey to touch the screen with one hand. A computer recorded the position and accuracy of each touch. It also controlled the presentation via the monitor of visual stimuli (colored shapes), which served as visual targets (CORTEX software, NIMH Laboratory of Neuropsychology). Eye movements were monitored using an Iscan infrared system. Four visual target items (disks of $5 \mathrm{~mm}$ in diameter) were presented as shown in Figure $1 A$. A central white square served as fixation point (FP). The lever was displayed just below the FP.

Behavioral task. Two male rhesus monkeys were trained in the problem-solving task (PS task) (Fig. 1 A,B). Monkeys had to find by trial and error which target, presented in a set of four, was rewarded. Each block of trials (or problem) contained a search period (exploration) during which the animal was searching for the rewarded target and, after its discovery, a repetition period (exploitation) during which the correct response was repeated at least three times (Fig. $1 B$ ). Each trial started by the onset of a starting target referred to as the "lever." The animal had to start a trial by touching the lever and holding his touch. The FP appeared and the animal had to fixate it with his gaze. A delay period (2 s) followed, which was ended by the simultaneous onset of the four targets. At the FP offset, the animal made a saccade toward a target, fixated it $(0.5$ s), and then touched it following the GO signal. All targets switched off at the touch and a $0.6 \mathrm{~s}$ delay followed before the feedback was given. A reward (squirt of fruit juice) was simultaneously delivered for choosing the correct target (positive feedback). If the choice was incorrect, no reward was delivered (negative feedback, INC trials), and the monkey could select another target in the following trial and so on until the solution was discovered (search period). Each feedback was followed by an intertrial interval of at least $1500 \mathrm{~ms}$. Any break in fixation requirements resulted in trial cessation.

After discovery of the correct target (first correct trial, CO1), the animal was allowed to repeat the correct response (COR). In $90 \%$ of cases, after the third repetition, a red flashing signal (SCS, the four targets in red) indicated the start of a new problem (i.e., a search for a new correct target). In $10 \%$ of cases, the repetition lasted for 7 or 11 trials. The "search" periods included all incorrect trials up to the first correct one, and the "repetition" periods included all trials until completion of the required number of correct repetitions. Note, however, that in the current report we mainly focus on INC and COR trials comparing negative feedback in search and positive feedback in repetition.

Recordings. Monkeys were implanted with a head-restraining device, and a craniotomy was made based on stereotaxic coordinates to expose an aperture over the prefrontal cortex. A recording chamber was implanted with its center placed at stereotaxic coordinates AP +31 . Neuronal activity was recorded using epoxy-coated tungsten electrodes (1-4 $\mathrm{MOhm}$ at $1 \mathrm{kHz}$; FHC). One to four microelectrodes were placed in stainless steel microguide tubes and independently advanced into the cortex by a set of micromotors (Alpha-Omega Engineering). Signals were sampled at $13 \mathrm{kHz}$ resolution for single unit neuronal activity and at 781 $\mathrm{Hz}$ for LFPs. Recordings were referenced on the guide tubes in contact with the dura and containing the microelectrodes. Recordings sites in ACC covered an area extending over $\sim 6 \mathrm{~mm}$ (anterior to posterior), in the dorsal bank of the anterior cingulate sulcus, in a region anterior to $\mathrm{AP}+30$, and at depths superior to $4.5 \mathrm{~mm}$ from cortical surface. This corresponds to a region recorded in previous reports and in which feedback-related activity has been observed (Procyk et al., 2000; Ito et al., 2003; Amiez et al., 2006; Quilodran et al., 2008). This part of the anterior cingulate cortex lies at the same AP level as the supplementary eye field and includes part of, and goes anterior to, the rostral cingulate motor area as evaluated from previous publications (Shima et al., 1991; Ito et al., 2003). Recording sites in LPFC were located mostly on the posterior third of the principal sulcus (area 9/46 from Petrides and Pandya, 1999). Locations were confirmed by anatomical MRI and histology (Fig. 1C). Dual recordings were performed using a double ensemble of four microguides separated by $8 \mathrm{~mm}$. The medial ensemble was used to drive electrodes toward the ACC, and the lateral one to the LPFC. The $x / y$ positions of the 
ensembles were interdependent and were positioned on the same rostrocaudal plane.

Data analyses. LFP signals were evaluated with the software package for electrophysiological analysis (ELAN-Pack) developed at the laboratory Inserm U1028 laboratory (ex Inserm U821, http://u821.lyon.inserm.fr/ index_en.php). LFPs were visualized and analyzed in the form of time frequency analyses. The time frequency analyses contained the averaged power of each frequency ranging from 2 $\mathrm{Hz}$ to $150 \mathrm{~Hz}$ in $2 \mathrm{~Hz}$ steps across all the trials for the entire dataset. Data were analyzed in the time frequency domain by convolution with complex Gaussian Morlet's wavelets with a ratio $f / \delta_{f}$ of 7 . We applied a Blackman window $(=100)$ to eliminate border effects. Trials were aligned on feedback onset (reward or no reward). The average signal at all frequencies taken from $-500 \mathrm{~ms}$ to $-300 \mathrm{~ms}$ before INC or COR feedback was subtracted (common baseline) from the corresponding data. This baseline appeared to be the more neutral period in terms of power variations for the gamma band. An example of normalized time frequency data aligned on the negative feedback onset is shown in Figure $2 A$.

Recordings at single recording sites were analyzed independently and selected for having correct recording quality (regarding electrical noise in particular). We evaluated postfeedback high gamma activity increases within $60-$ $100 \mathrm{~Hz}$ and $100-140 \mathrm{~Hz}$ bands using time frequency data for three types of feedback: INC, CO1, and COR. The two bands in higher frequencies were separated to improve signal detection. Indeed, as shown in Figure 2, some sites revealed gamma activity in a subzone of the high gamma band; averaging over the entire $60-140 \mathrm{~Hz}$ window might have increased false negative detections. The different foci of high gamma power in the range $60-140 \mathrm{~Hz}$ are frequently observed and might correspond to spatially dissociated neural populations (Sirota et al., 2008). To detect significant changes in gamma activity, we used a Wilcoxon test comparing the gamma activity around feedback onset with the gamma activity measured in the baseline ( -500 to $-300 \mathrm{~ms}$ before feedback). We used moving windows of $100 \mathrm{~ms}$ by $50 \mathrm{~ms}$ steps with a frequency resolution of $2 \mathrm{~Hz}$. A false detection rate (FDR) correction was then applied. This allowed us to compute the FDR statistics after the Wilcoxon test. This is a possible solution for multiple testing problems (Genovese et al., 2002). The latency of feedback-related gamma activity was taken as the time around the feedback onset at which the FDR data reached significance (Fig. $2 B$ ), that is, when the averaged corrected statistical $Z$ value $(>3$ or $<-3$ ) of the considered frequency band differed from 0 . To be considered significant, the gamma activity had to reach the threshold during at least $120 \mathrm{~ms}$. The distributions of gamma activity latencies observed in ACC and LPFC were compared with a Kruskal-Wallis test.

We analyzed the shape of the FDR-isolated clouds of activation after negative feedback (Fig. $2 B$ ) in terms of time duration, extent in fre-
A

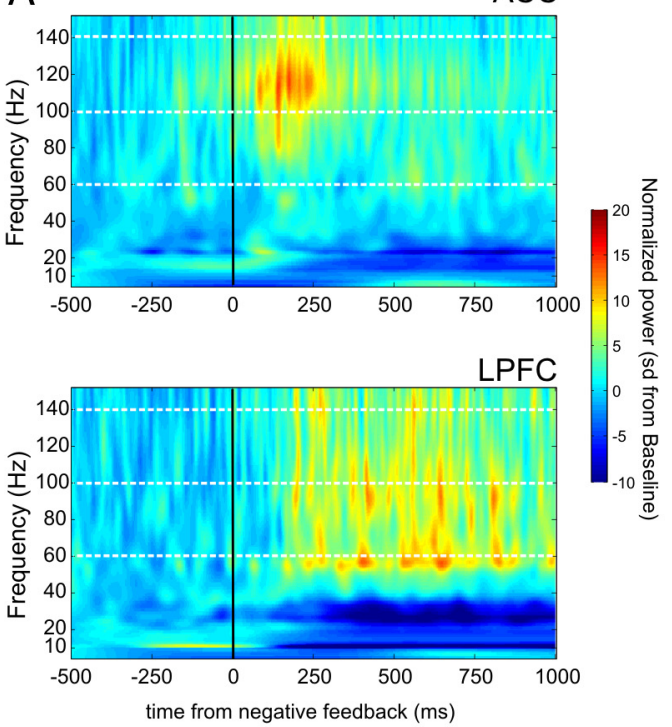

B

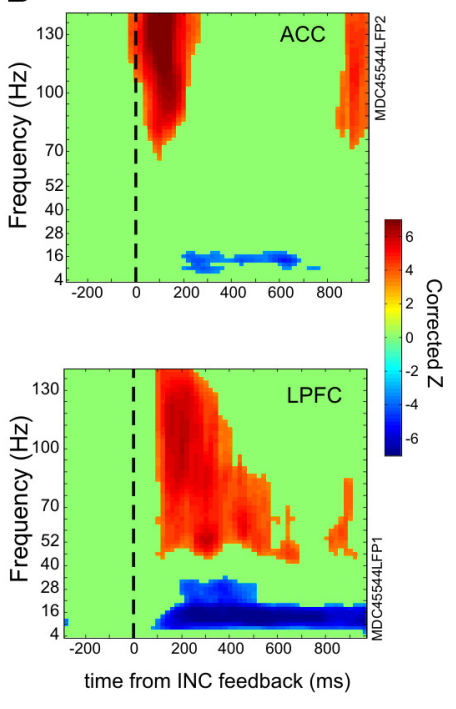

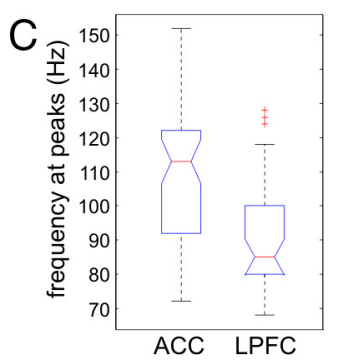

D

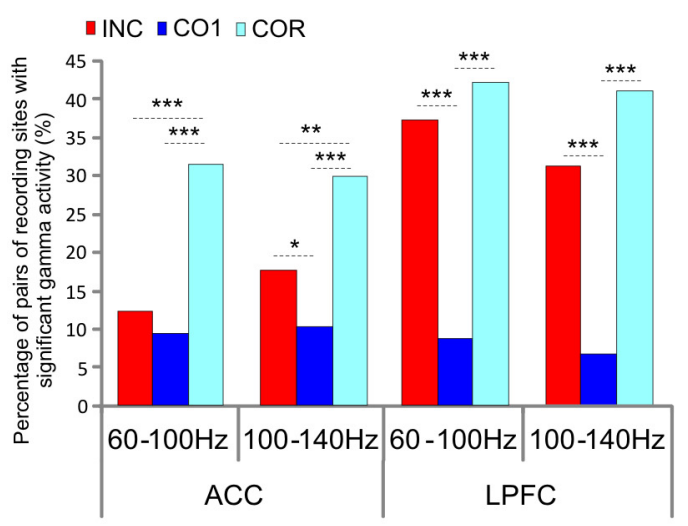

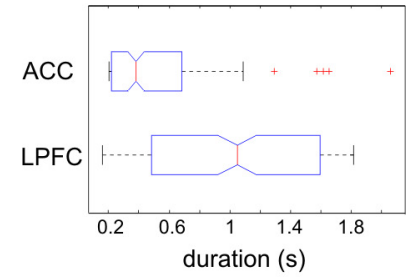

E
$\mathrm{ACC}$
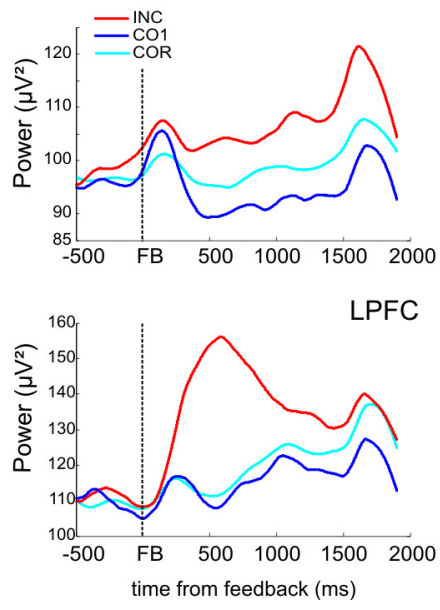

Figure 2. General description of high gamma oscillations. $A$, Average normalized power from 20 to $140 \mathrm{~Hz}$ from pairs of ACC and LPFC sites simultaneously recorded during incorrect trials. $\mathrm{ACC}, n=14 ; \mathrm{LPFC}, n=7 . \boldsymbol{B}$, Example of time frequency graph showing statistical change in activity compared with the baseline period ( $-500 /-300 \mathrm{~ms}$ ) before feedback (Wilcoxon and FDR). C, Boxplot of the frequency at peaks (left) and of the duration (right) of high gamma activations for recorded sites in ACC and LPFC after errors. The boxplots present the median (in red), the comparison intervals (notches), outliers (red crosses), box limits represent 25 th and 75 th percentiles, and whiskers give the $+/-2.7 \sigma$ interval. $D$, Proportion of sites with significant gamma power increase after feedback per frequency band. ACC: monkey M, $n=183$ sites; monkey $\mathrm{P}, n=61$ sites; LPFC: monkey $\mathrm{M}, n=76$ sites; monkey $\mathrm{P}, n=26$ sites. Data are presented for two gamma bands: $60-100 \mathrm{~Hz}$ and $100-140 \mathrm{~Hz}$. We observed more active sites in the repetition period than in the search period for the ACC and the same tendency in the LPFC for the INC and COR comparison. Activity linked to the $\mathrm{C} 01$ was significantly less present in both regions for both frequency bands. The asterisks indicates the significance level: ${ }^{*} p<0.05,{ }^{* *} p<0.01,{ }^{* * *} p<0.001$. E, Average raw activity $\left(\mu V^{2}\right)$ smoothed (rloess, 0.1 ) in the 100-140 Hz frequency band for the ACC (top, $n=244$ ) and LPFC (bottom, $n=102$ ) aligned on the three types of feedback (INC, red; CO1, light blue; COR, dark blue).

quency, and in terms of the frequency of peak activations (focal maxima in the cloud). An FDR-isolated cloud was defined as the first group of significant points in the FDR-corrected time frequency matrix that lasted at least $120 \mathrm{~ms}$. 

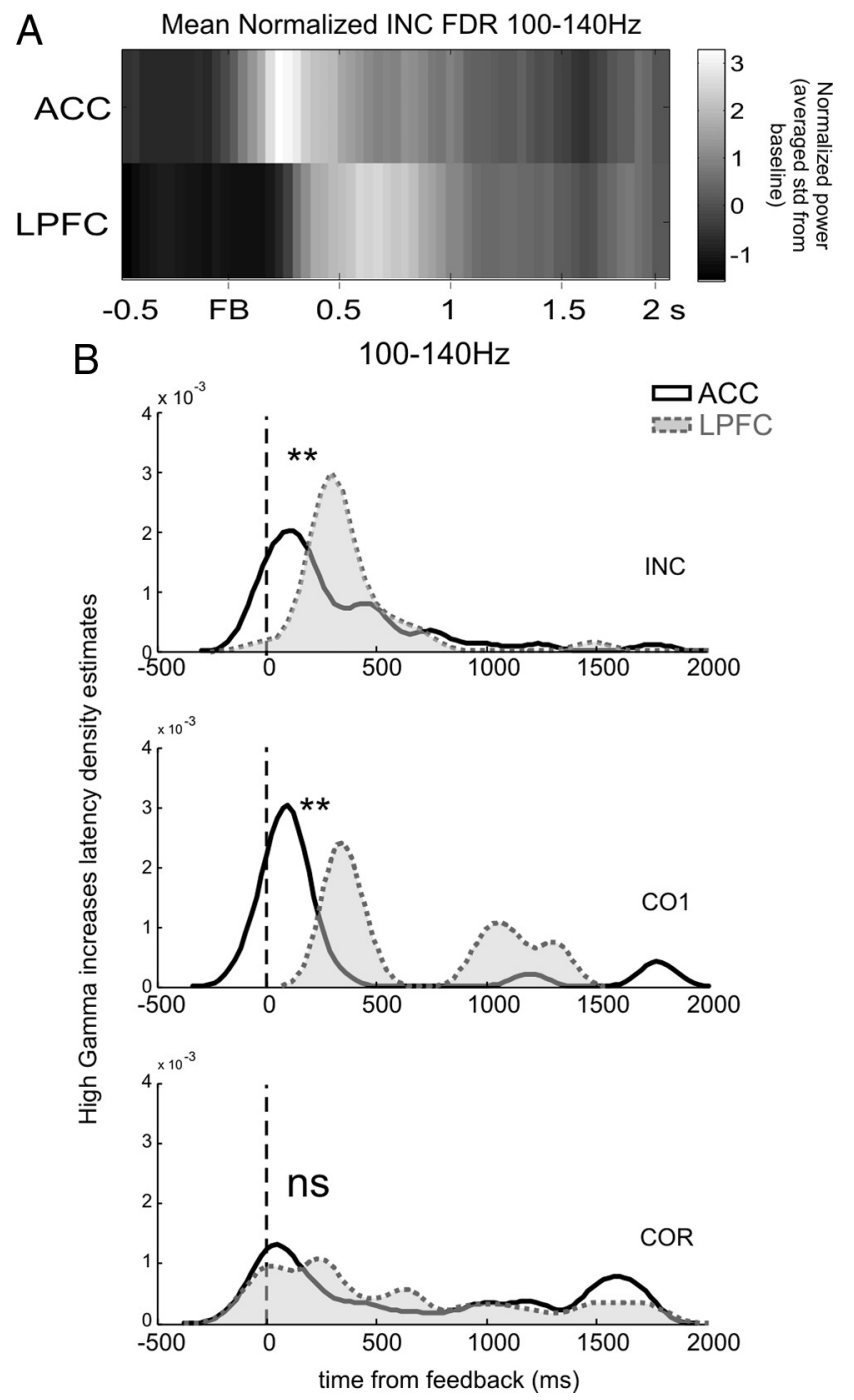

Figure 3. High gamma latencies after negative feedback (FB). $A$, Averaged significant $100-$ $140 \mathrm{~Hz}$ gamma per sites aligned on feedback delivery. The graph shows the normalized change in power of high gamma oscillations for INC feedback for the ACC (top) and the LPFC (bottom) compared with the baseline period. $\boldsymbol{B}$, Kernel density estimates of latency distributions. Populations of high gamma activation latencies for both regions revealed an overall advance of ACC over LPFC in search periods.

Linear correlations between the gamma power of ACC and LPFC were investigated in 47 pairs of simultaneously recorded sites in ACC and LPFC (monkey M: 14 pairs; monkey P: 33 pairs). Correlations were tested on sliding windows of $300 \mathrm{~ms}$ by $100 \mathrm{~ms}$ steps from -300 to 1100 $\mathrm{ms}$ around feedback onset (12 intervals). Correlations were tested between the trial-by-trial high gamma power measured in the different temporal windows of the two regions (12 temporal intervals), giving a $12 \times 12$ matrix of correlations. The statistical threshold was corrected for the multiple testing and set at $0.05 / 12=0.0042$. We then counted the number of pairs of recordings showing significant high gamma power correlation in at least one of the $144(12 \times 12)$ time windows.

All comparisons of proportions were performed with a proportion comparison test (prop.test without Yates' continuity correction, $p<$ 0.05, R v2.11, R Foundation for Statistical Computing).

A drop in the $10-20 \mathrm{~Hz}$ (beta) power seemed frequently present after feedback; we thus applied the same analyses as those just described for the high gamma band on power decreases in this frequency band.

\section{Results}

During recordings, monkeys performed optimal searches (i.e., did not repeat incorrect trials) and optimal repetitions: average number of trials in search: $2.4 \pm 0.15$ trials in monkey $\mathrm{M}, 2.65 \pm$ 0.23 in monkey $\mathrm{P}$ (optimal performance is 2.5; Procyk and Goldman-Rakic, 2006); in repetition: $3.14 \pm 0.7$ trials in monkey M, $3.4 \pm 0.55$ in monkey P. Optimal performances have been described previously (Procyk et al., 2000; Procyk and GoldmanRakic, 2006).

\section{Feedback-related high gamma activity}

We first evaluated the whole population of recordings performed in ACC and LPFC. This concerned 244 sites in the ACC (monkey M: 183; monkey P: 61) and 102 from LPFC (monkey M: 76; monkey P: 26). Forty-five percent of the ACC data used here were taken from a former dataset (Quilodran et al., 2008). Note that most recordings were performed separately in the two structures. Forty-seven recording pairs of signals simultaneously recorded in the two cortical areas were also included in the analysis.

Significant feedback-related high gamma band activity as previously observed in the ACC was found in the LPFC. We focused on variations related to feedback onset for INC, CO1, and COR trials. The increased power was observed on large bands from 60 $\mathrm{Hz}$ to $140 \mathrm{~Hz}$ with various expansions (Fig. 2A). We observed that, on average, significant increases in high gamma activity after errors were shorter in ACC than in LPFC (Fig. 2C), which fits with the tendency for LPFC to display tonic activations, and that high gamma peaks were at slightly higher frequencies for ACC than LPFC (Kruskal-Wallis, duration: $p=0.0021$; frequency peak: $\left.p=3.02 \times 10^{-5}\right)$.

In a first step, we evaluated the proportion of significantly activated sites (after feedback) for $60-100 \mathrm{~Hz}$ and $100-140 \mathrm{~Hz}$. Results from both frequency bands were comparable: $12.3 \%$ and $17.6 \%$ of ACC recording sites (for $60-100 \mathrm{~Hz}, n=30 / 244$, and 100-140 Hz, $n=43 / 244$, respectively) showed significant feedback-related increases in high gamma bands after errors (INC), compared with $9.4 \%$ and $10.2 \%$ of ACC recording sites for CO1 (for $60-100 \mathrm{~Hz}, n=23 / 244$, and $100-140 \mathrm{~Hz}, n=$ $25 / 244$, respectively), and $31.6 \%$ and $29.9 \%$ after correct (COR) in repetition $(60-100 \mathrm{~Hz}, n=77 / 244$, and $100-140 \mathrm{~Hz}, n=$ $73 / 244$, respectively). The proportion of sites with significant gamma power increases for correct in repetition was significantly larger than for errors in search periods in ACC and for both frequency bands (Fig. 2D) (for 60-100 Hz: INC/COR, $\chi^{2}=25.3$, $p=4.8 \times 10^{-7}$; CO1/COR, $\chi^{2}=35.3, p=2.8 \times 10^{-9}$; INC/ CO1, n.s.; for the 100-140 Hz band: INC/COR, $\chi^{2}=9.51, p=$ 0.002043; CO1/COR, $\chi^{2}=28.2, p=1.09 \times 10^{-7}$; INC/CO1, $\left.\chi^{2}=4.9, p=0.026\right)$. In a previous report, we compared site by site the level of postfeedback activity in search and repetition periods (Quilodran et al., 2008). We found that ACC sites with higher feedback-related activity in search than in repetition were more frequent than the reverse. This is reflected here in the grand average raw signal (from all recording sites) showing a large dominant increase in power after incorrect feedback (Fig. 2E). Note that increased gamma for COR trials occurred, in particular, at the end of the intertrial interval. To conclude, high gamma power increases were more frequently observed after COR than after INC feedback but differed in power and temporal patterns. This suggests changes in the contribution and dynamic of the two regions between the two behavioral periods of the task.

For LPFC recordings, feedback-related increases after errors were found in $37.3 \%$ and $31.4 \%$ (for $60-100 \mathrm{~Hz}, n=38 / 102$, and $100-140 \mathrm{~Hz}, n=32 / 102$, respectively) of recordings sites (Fig. $2 \mathrm{D})$, compared with $8.8 \%$ and $6.9 \%$ for CO1 $(60-100 \mathrm{~Hz}, n=$ $9 / 244$, and $100-140 \mathrm{~Hz}, n=7 / 244$, respectively) and $42.2 \%$ and $41.2 \%(n=43 / 102 ; n=42 / 102)$ after correct in repetition. In- 
creases after $\mathrm{CO} 1$ feedback were significantly less frequent for both frequency bands (for 60-100 Hz: INC/CO1, $\chi^{2}=21.67$, $p=3.2 \times 10^{-6}$; CO1/COR, $\chi^{2}=28.1, p=1.1 \times 10^{-7}$; INC/ COR, n.s.; and for 100-140 Hz: INC/CO1, $\chi^{2}=18.3, p=1.9 \times$ $10^{-5}$; CO1/COR, $\chi^{2}=31, p=2.5 \times 10^{-8}$; INC/COR, n.s.). INC-related activities were less present than COR-related ones, although the difference in proportions was not significant in this cortical area.

In summary, ACC revealed more frequent feedback-related high gamma responses after positive feedback in repetition than after feedback in search, and LPFC revealed only a nonsignificant tendency. However, as clearly illustrated in Figure 2D, early and strong power increases took place after negative feedback, whereas increases in repetition were observed later in the postfeedback period in particular for LPFC.

\section{Distribution of high gamma latencies in the ACC and LPFC}

One major question concerned the possible sequential activation of ACC and LPFC in response to negative feedback, as predicted by models of cognitive control. We first investigated whether the latencies of high gamma power increases after the errors in the search period were different in the two structures. Errors usually imply a need for subsequent adaptation and thus should lead to the strongest interactions between ACC and LPFC. Positive feedback in our task implies a stay strategy, which could lead to different interactions between the two cortical regions.

Latencies were computed by detecting the time point at which the high gamma power reached the statistical threshold for at least $120 \mathrm{~ms}$ (see Materials and Methods). We compared the overall populations of latencies for recordings in ACC and LPFC. Note that our method for investigating latencies of activity was sensitive to any activity occurring around feedback including prefeedback anticipatory (from $-300 \mathrm{~ms}$ ) and late postfeedback activity (to $+1100 \mathrm{~ms}$ ) that could correspond to late processing of feedback-related activity and intertrial adjustments.

To represent the dynamical pattern of activity increases in both structures, we produced the averaged normalized high gamma power modulations (in standard deviation from average total activity: -3 to +2 s around feedback onset) curves for both regions. This clearly revealed that increases in power after errors occurred earlier for ACC sites than for LPFC sites (Fig. 3A). In other words, power increases revealed a time lag in activity after errors between ACC and LPFC.

The latency distributions for high gamma power increases were significantly different between ACC and LPFC, with an advance of ACC activity over LPFC for feedback in search only (Kruskal-Wallis test, 60-100 Hz: INC, $p=0.0015$; CO1, $p=$ 0.009; COR, $p=0.6$; $100-140 \mathrm{~Hz}$ : INC, $p=0.0177$; CO1, $p=$ 0.0023 ; COR, $p=0.6$ ) (Fig. $3 B$ ). Thus, high gamma power increases reached significance earlier in ACC than in LPFC during the search period. Interestingly, the lag between ACC and LPFC disappeared for COR feedback, revealing a sharp change between search and repetition periods. Activation latencies were more spread along the postfeedback interval during the repetition period, with less clear temporal clustering of latencies. This was related in particular to increased gamma occurring $>1000$ $\mathrm{ms}$ after the feedback in anticipation of the next trial initiation. In conclusion, the latency distributions revealed that the coordination of ACC and LPFC activations changed between the two behavioral periods, with a clear lag between structures in the period requiring behavioral adaptation.
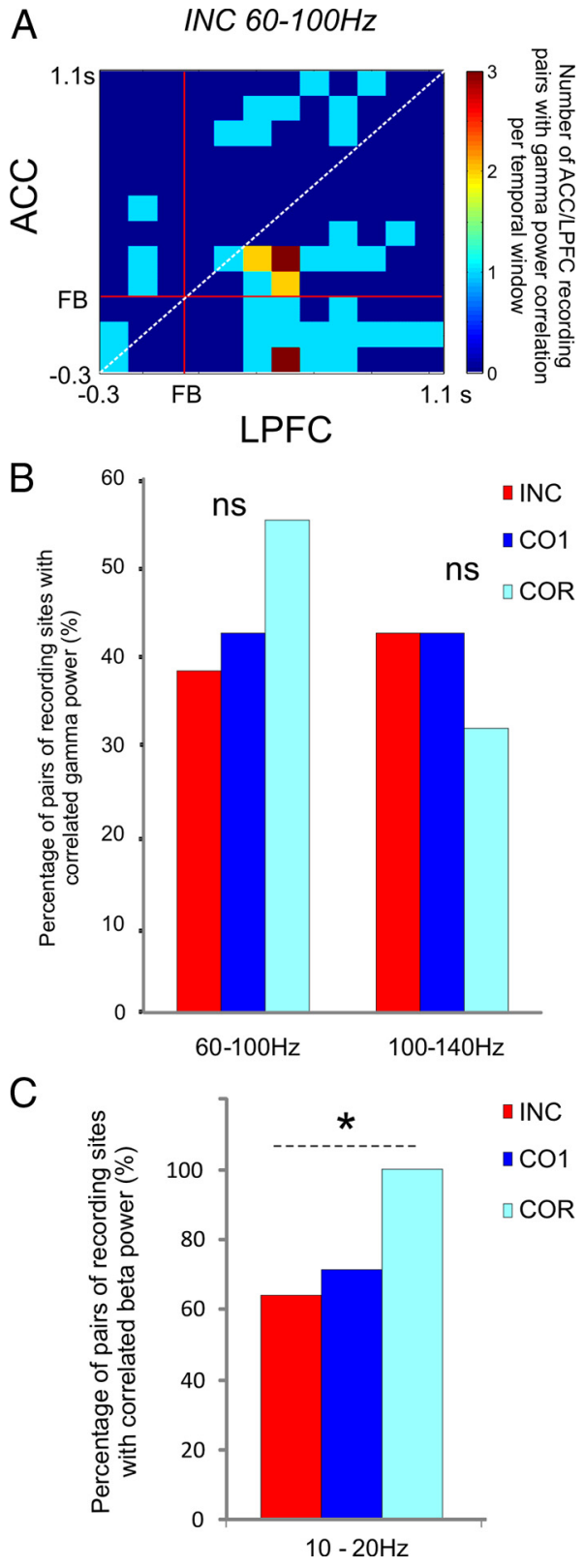

Figure 4. High gamma and beta power correlations after feedback. $A$, Temporal distribution of significant power correlations for search trials. For $60-100 \mathrm{~Hz}$, we observed in the search period only a tendency for early ACC power to correlate with later LPFC power. $B$, Percentage of ACC/LPFC simultaneous recordings with gamma power correlation after INC, CO1, and COR in at least one $300 \mathrm{~ms}$ interval from $-300 \mathrm{~ms}$ to $1100 \mathrm{~ms}$ (see Materials and Methods for details). C, Percentage of ACC/LPFC simultaneous recordings with beta power correlation after INC, CO1, and $C O R$ in at least one $500 \mathrm{~ms}$ interval from $-500 \mathrm{~ms}$ to $1000 \mathrm{~ms}$ (see Materials and Methods for details). We observed more frequent correlations in the repetition period than in the search period.

\section{Correlations of high gamma activity between the ACC and the LPFC}

Given the profiles of high gamma activity in the ACC and in the LPFC after errors (Figs. $2 A, 3 A$ ), we wondered whether a quantitative link could be demonstrated between the gamma power measured in the two regions. We tested the presence of trial-bytrial power correlations between ACC and LPFC sites recorded simultaneously. Using sliding windows of $300 \mathrm{~ms}$ from $-300 \mathrm{~ms}$ to $1100 \mathrm{~ms}$ around the feedback with $100 \mathrm{~ms}$ steps (see Materials and Methods and Fig. $4 A$ ), we found for INC trials that $38.3 \%$ 

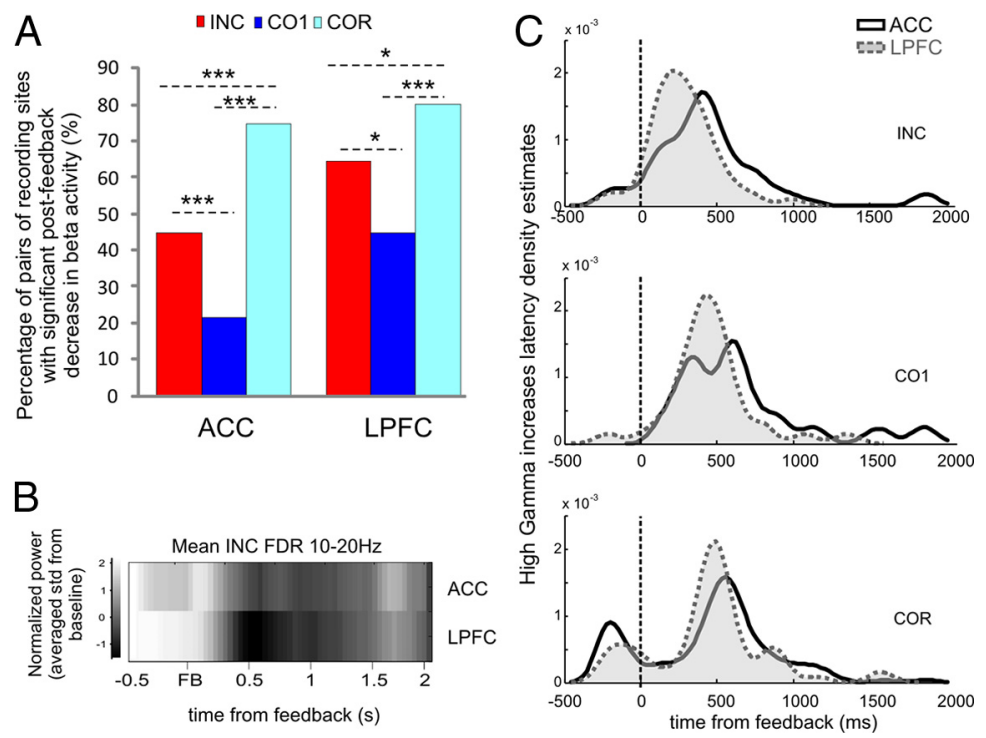

Figure 5. Beta band analyses. $A$, Percentage of sites with beta power desynchronization in the ACC (left) and LPFC (right). We observed more activity during the repetition period compared with the search period. $\boldsymbol{B}$, Average of significant beta per sites aligned on feedback delivery. The graph shows the normalized averaged change in power of high beta oscillations for INC feedback for the ACC (top) and the LPFC (bottom) compared with the baseline period. C, Kernel density estimate. Cumulative proportions of beta deactivation latencies for both regions revealed an overall advance of LPFC over ACC for search trials only.

and $42.6 \%$ of ACC and LPFC sites, respectively (18/47 pairs and $20 / 47$ pairs for $60-100 \mathrm{~Hz}$ and $100-140 \mathrm{~Hz}$, respectively) contained at least one time window with correlated high gamma activity. The proportion was $42.6 \%$ (20/47 pairs for both frequency bands) for CO1 trials, whereas $55.3 \%$ and $31.9 \%$ of pairs (26/47 pairs for $60-100 \mathrm{~Hz}$ and $15 / 47$ pairs for $100-140 \mathrm{~Hz}$ ) showed correlations for COR trials (Fig. $4 B$ ). To test whether correlations within correlation matrices of the different pairs overlapped in time, we examined the occurrences of correlations over all pairs. This revealed only weak, if any, overlap for search trials (INC and CO1). We observed, for the $60-100 \mathrm{~Hz}$ in the search period, a tendency for early ACC power to correlate with later LPFC power (Fig. 4A); however, this phenomenon was not observed for the repetition period or for the 100-140 Hz gamma band.

\section{Beta frequency band activity in the ACC and the LPFC}

In one animal (monkey $\mathrm{M}$ ), we repeatedly observed elevated beta oscillations power before feedback followed by a sharp desynchronization after feedback. A large proportion of recording sites contained decreases in the beta band power after feedback. Beta decreases were more frequent after feedback in the repetition period compared with the search period in the ACC and in the LPFC [ACC: INC, 82/183 (44.8\%); CO1, 39/183 (21.3\%); COR, 137/183 (74.9\%). LPFC: INC, 49/76 (64.5\%), CO1, 34/76 (44.7\%); COR, $61 / 76$ (80.3\%). ACC: INC/COR, $\chi^{2}=33.2, p=$ $8.5 \times 10^{-9} ; \mathrm{CO} 1 / \mathrm{COR}, \chi^{2}=102.9813, p=2.2 \times 10^{-16} ; \mathrm{INC} / \mathrm{CO} 1$, $\chi^{2}=21.77, p=3.06 \times 10^{-6}$. LPFC: INC/COR, $\chi^{2}=3.98, p=$ 0.046 ; CO1/COR, $\chi^{2}=18.97, p=1.324 \times 10^{-5}$; INC/CO1, $\chi^{2}=$ 5.20, $p=0.02256$ ] (Fig. 5A).

Beta desynchronization latencies were significantly different between ACC and LPFC for incorrect (Fig. 5B) and first correct trials of the search period (Kruskal-Wallis test, INC, $p=0.0027$; CO1, $p=0.03$; COR, $p=0.2857$ ) with an earlier LPFC desynchronization compared with ACC (Fig. 5C).

We tested whether power correlations took place between ACC and LPFC in $10-20 \mathrm{~Hz}$ and obtained positive results for both periods of the task (INC, $n=9 / 14$; CO1, $n=10 / 14$; COR, $n=14 / 14)$. Proportions were significantly different between repetition (COR) and negative feedback in search $\left(\chi^{2}=3.89, \mathrm{df}=1, p=\right.$ 0.048 ) but not between COR and CO1 (Fig. 4C).

In conclusion, as for high gamma power, beta activity revealed a lag between ACC and LPFC only for the search period. The correlations of beta power were more frequent in trials of the repetition period compared with incorrect trials of the search period. This also suggests different modes of coordination between ACC and LPFC depending on the behavioral period of the problem-solving task.

\section{Discussion}

We focused on high gamma LFP (HG) recorded in ACC and LPFC to test three specific predictions: (1) ACC and LPFC should show increased activity after outcomes indicating adaptation; (2) in that case, ACC feedback-related activity should precede LPFC; and (3) simultaneous gamma power recorded in both structures is correlated. We found that ACC shows advanced activation over LPFC after feedback of the search period, but that there is no systematic lag between the activations in the two cortical areas during repetition. In contrast, correlations of high gamma activity were present for both search and repetition periods. In addition, beta oscillation power decreased after feedback onset in the LPFC, followed by a decrease in the ACC exclusively during the search period. Correlations of beta power from the two structures were predominant during repetition. Those results suggest that the coordination between ACC and LPFC follows two different modes depending on the task requirements.

HG is considered as a good marker of local neural processing. Power in gamma bands contains task-related information and can be used to study functional specificity in local areas (Pesaran et al., 2002; Lachaux et al., 2007; Belitski et al., 2008). We observed that peaks of activation were located at various frequencies and with various extents in the two structures. This confirms descriptions in rodent cortex, where gamma activity with particular localization and/or frequency peaks were found to be associated with the activity of unique groups of neurons, were transient, and were highly localized (Sirota et al., 2008). As previously reported with various preparations, HG is usually negatively correlated with beta oscillations. As shown here for the two frontal cortical areas, a reduction of beta power is frequently observed in parallel with an increase in HG (Buehlmann and Deco, 2008; Ray et al., 2008). HG has been shown to correlate positively with fMRI BOLD signal and with local coupling of single spike activity, whereas beta power is negatively correlated with BOLD signal (Scheeringa et al., 2011). Those two frequencies seem to contribute independently to the activated state of cortical areas.

Our previous report showed that ACC feedback-related gamma activity was prominent for incorrect and first correct trials in search (Quilodran et al., 2008). The results presented here show that both cortical areas have a particular role in encoding outcomes or its consequences during the exploration phase of 
the trial and error task. This is in line with the recurring observations of $\mathrm{fMRI}$ BOLD increases in equivalent areas during cognitively demanding tasks in humans (Duncan and Owen, 2000). ACC activity measured in high gamma appears transient at negative feedback compared with LPFC. This is coherent with past descriptions of single unit activity that is highly related to feedback evaluation in ACC (Quilodran et al., 2008) and of tonic activity patterns observed in LPFC and likely related to prospective planning (Constantinidis and Procyk, 2004; Procyk and Goldman-Rakic, 2006). However, high levels of activity after errors might also reflect global changes in cortical functioning. Previous works indeed have revealed changing modes of activations in ACC and LPFC at transitions for exploration to repetition periods (Procyk et al., 2000; Procyk and Goldman-Rakic, 2006).

One simple prediction to draw from cognitive control loop models is that ACC performance-monitoring signals should occur before LPFC cognitive control-related activity because the former is supposed to trigger the latter. A temporal segregation between ACC and LPFC was not found in monkeys using an executive control-challenging task, characterized by visual cues and probes, and analyzing local field potentials (Dias et al., 2006). We reasoned that during the problem-solving task, which does not provide explicit visual information, negative feedback in search is likely to drive self-organized performance adjustment and hence measurable functional coordination between ACC and LPFC. Both structures showed increased high gamma oscillations after errors, with a lag in activity latencies placing ACC activations before LPFC. However, the observed average latency difference surpassed $100 \mathrm{~ms}$, a difference larger than what would be expected from direct corticocortical interactions. Different interpretations could be made of such an unexpected difference, including biases in cortical sites sampling. These results could also reveal that the functional relationship between ACC and LPFC is not direct. Aston-Jones and Cohen (2005) proposed that the noradrenergic system would be an intermediate by which ACC outputs regulate cortical processing and in particular LPFC. The latency could also in part emerge from the properties of ACC to LPFC synaptic connections engaged during adaptation. Medalla and Barbas $(2009,2010)$ recently suggested that a subregion of the ACC (area 32) would act by influencing inhibitory interneurons in area 46 and hence the signal-to-noise ratio, instead of acting through a feedforward transfer of information. One could speculate that if a direct link between ACC and LPFC was involved, then a modulatory effect could contribute to an increase in latencies.

The dynamic of activity within the performance monitoring network is likely to be more complex than a simple one-way causal relationship between ACC and LPFC. Unit activity in the supplementary eye field also reacts to errors with latencies shorter than in ACC (Ito et al., 2003; Emeric et al., 2008). Thus, other cortical areas might be involved in error processing with different temporal dynamics. Note, however, that those latter studies measured error-related activity in a countermanding saccade task that might particularly reveal motor-related error signals as opposed to performance feedback.

A second possible prediction from cognitive control loop models is that the amplitude of cognitive control (supported by LPFC) correlates with the amplitude of performance-monitoring signals within ACC. In most computational models, prediction error signals or conflict-monitoring outputs directly scale cognitive control (Botvinick et al., 2001; Brown and Braver, 2005). These hypotheses come in part from behavioral data suggesting that changes in cognitive control are gradual (Botvinick et al., 2001). We addressed this question by looking at correlation of high gamma oscillation power in ACC and LPFC after errors and successes. This was motivated by the fact that gamma oscillation power could accompany the increased activity and synchrony of unit activity devoted to cognitive control. Indeed, LPFC spikes show synchronization during working memory tasks (Funahashi and Inoue, 2000; Constantinidis et al., 2001). In addition, correlation of high gamma power, but not phase locking, has been found between distant areas in human recordings (Lachaux et al., 2005). Sliding window correlation analyses were performed on simultaneous recordings in the two areas. More than $40 \%$ of pairs revealed power correlations between the distant LFP gamma power for negative feedback, but similar proportions of correlated pairs were also found for feedback during repetitions. Thus, HG power correlations appeared between the two areas but were not different between the two behavioral periods in our experiment. The absence of clear differential patterns of power correlation could be due to an insufficient number of pairs tested, or to the fact that the variations in interaction between the two periods is to be found in other types of neural relations. Our data suggest that a direct functional interaction between ACC and LFPC is unlikely to be clearly identified using high gamma power correlations.

HG power correlations were present for both INC and COR trials. This contrasts with the temporal organization of the local neural synchronizations (high gamma power) occurring only after INC and CO1 of the search period. Overall, these results reveal that two different mechanisms and possibly two different modes of coordination might take place during search and repetition periods. In their 2010 review, Engel and Fries suggested that maintenance of stable behavior is linked with interactions in the beta band between early and higher order areas, whereas gamma interactions would occur in relation to changes in the environment. The occurrence of temporal organization of high gamma power increases during the search period and the predominance of beta power correlations during the repetition period would support this hypothesis. The lower frequency band activity would allow the maintenance of a routine-like situation in the repetition period, whereas higher frequency band activity would reflect network interactions specific for flexible behaviors. Latency data from the exploration (search) periods support the ACC to LPFC relationship during adaptation; however, our data do not provide evidence that ACC and LPFC interact directly and in a specific manner during adaptation.

Different subdivisions of ACC send to and receive from LPFC direct anatomical connections (Sallet et al., 2011). Understanding the relative contribution of this direct connectivity compared with more global coordination of functions within large-scale networks will be an important challenge. Several problems will need to be addressed to investigate further the relationships between medial and lateral frontal cortical areas. First, the sites of recordings selected in each area might be of importance. The mechanisms by which complementary information is shared between two points of recording might depend on the type of corticocortical connections that exists between the lateral and medial subdivisions that are simultaneously recorded. Unpublished anatomical investigations from our institute have shown that the type (feedforward/feedback) and strength of connectivity depend on which part of ACC or LPFC is concerned (Quilodran et al., 2010). Second, the type and direction of interactions could evolve and change over short time scales after feedback, from simultaneous recurrent activation to sequential information processing. Note that such changing interactions could hardly be 
evidenced with variations in BOLD signals or with latencies of power increases. Specific analyses of unit and LFP should address these issues in the future.

\section{References}

Amiez C, Joseph JP, Procyk E (2006) Reward encoding in the monkey anterior cingulate cortex. Cereb Cortex 16:1040-1055.

Aston-Jones G, Cohen JD (2005) An integrative theory of locus coeruleusnorepinephrine function: adaptive gain and optimal performance. Annu Rev Neurosci 28:403-450.

Averbeck BB, Chafee MV, Crowe DA, Georgopoulos AP (2002) Parallel processing of serial movements in prefrontal cortex. Proc Natl Acad Sci U S A 99:13172-13177.

Averbeck BB, Sohn JW, Lee D (2006) Activity in prefrontal cortex during dynamic selection of action sequences. Nat Neurosci 9:276-282.

Belitski A, Gretton A, Magri C, Murayama Y, Montemurro MA, Logothetis NK, Panzeri S (2008) Low-frequency local field potentials and spikes in primary visual cortex convey independent visual information. J Neurosci 28:5696-5709.

Botvinick MM, Braver TS, Barch DM, Carter CS, Cohen JD (2001) Conflict monitoring and cognitive control. Psychol Rev 108:624-652.

Brown JW, Braver TS (2005) Learned predictions of error likelihood in the anterior cingulate cortex. Science 307:1118-1121.

Buehlmann A, Deco G (2008) The neuronal basis of attention: rate versus synchronization modulation. J Neurosci 28:7679-7686.

Cavanagh JF, Cohen MX, Allen JJ (2009) Prelude to and resolution of an error: EEG phase synchrony reveals cognitive control dynamics during action monitoring. J Neurosci 29:98-105.

Constantinidis C, Procyk E (2004) The primate working memory networks. Cogn Affect Behav Neurosci 4:444-465.

Constantinidis C, Franowicz MN, Goldman-Rakic PS (2001) Coding specificity in cortical microcircuits: a multiple-electrode analysis of primate prefrontal cortex. J Neurosci 21:3646-3655.

Dehaene S, Kerszberg M, Changeux JP (1998) A neuronal model of a global workspace in effortful cognitive tasks. Proc Natl Acad Sci U S A 95:14529-14534

Dias EC, McGinnis T, Smiley JF, Foxe JJ, Schroeder CE, Javitt DC (2006) Changing plans: neural correlates of executive control in monkey and human frontal cortex. Exp Brain Res 174:279-291.

Duncan J, Owen AM (2000) Common regions of the human frontal lobe recruited by diverse cognitive demands. Trends Neurosci 23:475-483.

Emeric EE, Brown JW, Leslie M, Pouget P, Stuphorn V, Schall JD (2008) Performance monitoring local field potentials in the medial frontal cortex of primates: anterior cingulate cortex. J Neurophysiol 99:759-772.

Engel AK, Fries P (2010) Beta-band oscillations-signalling the status quo? Curr Opin Neurobiol 20:156-165.

Fassbender C, Hester R, Murphy K, Foxe JJ, Foxe DM, Garavan H (2009) Prefrontal and midline interactions mediating behavioural control. Eur J Neurosci 29:181-187.

Funahashi S, Inoue M (2000) Neuronal interactions related to working memory processes in the primate prefrontal cortex revealed by crosscorrelation analysis. Cereb Cortex 10:535-551.

Genovese CR, Lazar NA, Nichols T (2002) Thresholding of statistical maps in functional neuroimaging using the false discovery rate. Neuroimage 15:870-878.

Hikosaka O, Isoda M (2010) Switching from automatic to controlled behavior: cortico-basal ganglia mechanisms. Trends Cogn Sci 14:154-161.

Hyafil A, Summerfield C, Koechlin E (2009) Two mechanisms for task switching in the prefrontal cortex. J Neurosci 29:5135-5142.

Ito S, Stuphorn V, Brown JW, Schall JD (2003) Performance monitoring by the anterior cingulate cortex during saccade countermanding. Science 302:120-122.

Kennerley SW, Dahmubed AF, Lara AH, Wallis JD (2009) Neurons in the frontal lobe encode the value of multiple decision variables. J Cogn Neurosci 21:1162-1178.

Kerns JG, Cohen JD, MacDonald AW 3rd, Cho RY, Stenger VA, Carter CS (2004) Anterior cingulate conflict monitoring and adjustments in control. Science 303:1023-1026.
Koechlin E, Hyafil A (2007) Anterior prefrontal function and the limits of human decision-making. Science 318:594-598.

Lachaux JP, George N, Tallon-Baudry C, Martinerie J, Hugueville L, Minotti L, Kahane P, Renault B (2005) The many faces of the gamma band response to complex visual stimuli. Neuroimage 25:491-501.

Lachaux JP, Fonlupt P, Kahane P, Minotti L, Hoffmann D, Bertrand O, Baciu M (2007) Relationship between task-related gamma oscillations and BOLD signal: new insights from combined fMRI and intracranial EEG. Hum Brain Mapp 28:1368-1375.

Landmann C, Dehaene S, Pappata S, Jobert A, Bottlaender M, Roumenov D, Le Bihan D (2007) Dynamics of prefrontal and cingulate activity during a reward-based logical deduction task. Cereb Cortex 17:749-759.

Matsumoto K, Suzuki W, Tanaka K (2003) Neuronal correlates of goalbased motor selection in the prefrontal cortex. Science 301:229-232.

Medalla M, Barbas H (2009) Synapses with inhibitory neurons differentiate anterior cingulate from dorsolateral prefrontal pathways associated with cognitive control. Neuron 61:609-620.

Medalla M, Barbas H (2010) Anterior cingulate synapses in prefrontal areas 10 and 46 suggest differential influence in cognitive control. J Neurosci 30:16068-16081.

Pesaran B, Pezaris JS, Sahani M, Mitra PP, Andersen RA (2002) Temporal structure in neuronal activity during working memory in macaque parietal cortex. Nat Neurosci 5:805-811.

Petrides M, Pandya DN (1999) Dorsolateral prefrontal cortex: comparative cytoarchitectonic analysis in the human and the macaque brain and corticocortical connection patterns. Eur J Neurosci 11:1011-1036.

Procyk E, Goldman-Rakic PS (2006) Modulation of dorsolateral prefrontal delay activity during self-organized behavior. J Neurosci 26:11313-11323.

Procyk E, Tanaka YL, Joseph JP (2000) Anterior cingulate activity during routine and nonroutine sequential behaviors in macaques. Nat Neurosci 3:502-508.

Procyk E, Amiez C, Quilodran R, Joseph JP (2008) Modulations of prefrontal activity related to cognitive control and performance monitoring. In: Sensorimotor foundations of higher cognition attention and performance (attention and performance XXII) (Haggard P, Rossetti Y, Kawato M, eds), pp 27-46. Oxford, UK: Oxford UP.

Quilodran R, Rothé M, Procyk E (2008) Behavioral shifts and action valuation in the anterior cingulate cortex. Neuron 57:314-325.

Quilodran R, Sallet J, Procyk E, Kennedy H (2010) Heterogeneous connectivity patterns between anterior cingulate and lateral prefrontal cortices in the macaque monkey. In: 7th FENS Forum of European Neuroscience, The Netherlands, Amsterdam, FENS Abstr. vol 5, 088.49.

Ray S, Crone NE, Niebur E, Franaszczuk PJ, Hsiao SS (2008) Neural correlates of high-gamma oscillations $(60-200 \mathrm{~Hz})$ in macaque local field potentials and their potential implications in electrocorticography. J Neurosci 28:11526-11536.

Sallet J, Mars RB, Quilodran R, Procyk E, Petrides M, Rushworth MFS (2011) Neuroanatomical basis of motivational and cognitive control: a focus on the medial and lateral prefrontal cortex. In: Neural basis of motivational and cognitive control (Mars RB, Sallet J, Yeung N, Rushworth MF, eds). Cambridge: MIT.

Scheeringa R, Fries P, Petersson KM, Oostenveld R, Grothe I, Norris DG, Hagoort P, Bastiaansen MC (2011) Neuronal dynamics underlying high- and low-frequency EEG oscillations contribute independently to the human BOLD signal. Neuron 69:572-583.

Seo H, Lee D (2008) Cortical mechanisms for reinforcement learning in competitive games. Philos Trans R Soc Lond B Biol Sci 363:3845-3857.

Shima K, Aya K, Mushiake H, Inase M, Aizawa H, Tanji J (1991) Two movement-related foci in the primate cingulate cortex observed in signaltriggered and self-paced forelimb movements. J Neurophysiol 65:188-202.

Sirota A, Montgomery S, Fujisawa S, Isomura Y, Zugaro M, Buzsáki G (2008) Entrainment of neocortical neurons and gamma oscillations by the hippocampal theta rhythm. Neuron 60:683-697.

Tsujimoto T, Shimazu H, Isomura Y (2006) Direct recording of theta oscillations in primate prefrontal and anterior cingulate cortices. J Neurophysiol 95:2987-3000.

Wallis JD, Kennerley SW (2010) Heterogeneous reward signals in prefrontal cortex. Curr Opin Neurobiol 20:191-198. 